\title{
Technologic Papers
}

OF THE

\section{BUREAU OF STANDARDS}

S. W. STRATTON, DIRECTOR

No. 132

MECHANICAL PROPERTIES AND RESISTANCE TO CORROSION OF ROLLED LIGHT ALLOYS OF

ALUMINUM AND MAGNESIUM WITH

COPPER, WITH NICKEL, AND

WITH MANGANESE

BY

P. D. MERICA, Physicist

R. G. WALTENBERG, Assistant Physicist

and

A. N. FINN, Associate Chemist

Bureau of Standards

ISSUED OCTOBER 25, 1919

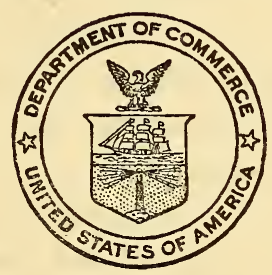

PRICE, 5 CENTS

Sold only by the Superintendent of Documents, Government Printing Office, Washington, D. C.

WASHINGTON

GOVERNMENT PRINTING OFFICE 


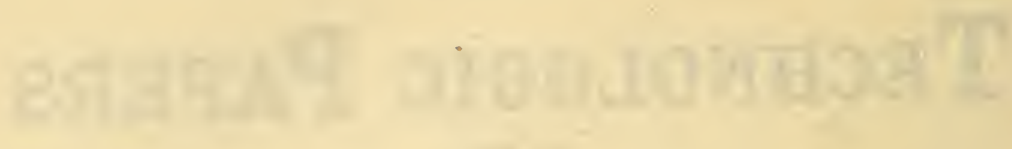

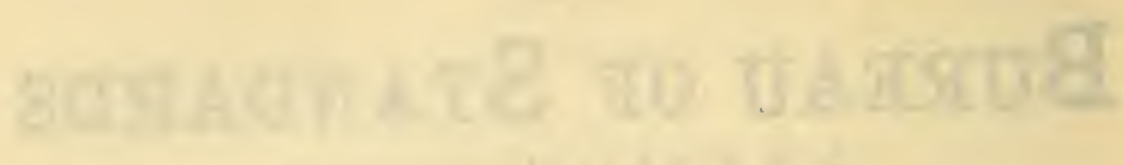

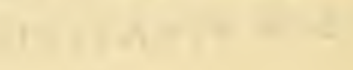

\section{$1+1$}

+

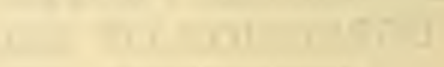

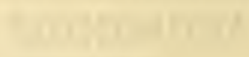




\title{
MECHANICAL PROPERTIES AND RESISTANCE TO CORROSION OF ROLLED LIGHT ALLOYS OF ALLUMINUM AND MAGNE- SIUM WITH COPPER, WITH NICKEL, AND WITH MANGANESE
}

\author{
By P. D. Merica, R. G. Waltenberg, and A. N. Finn
}

CONTENTS

Page.
3
4
5
5
9
12

\section{INTRODUCTION}

Certain compositions of the light, i. e., aluminum-rich, alloys of aluminum with magnesium and copper have become quite well known within the past ro years under the name of duralumin. These alloys are used for rolling and forging and can be so treated as to develop quite remarkable mechanical properties. Thus a properly heat treated alloy containing about 4 per cent of copper and about 0.5 per cent of magnesium, rolled into sheet or rod, will have a tensile strength of approximately 55 ooo pounds per square inch, with an elongation in 2 inches of about i 5 per cent. This alloy, duralumin, was developed by Wilm, ${ }^{1}$ and its properties more fully described by Cohn. ${ }^{2}$

The authors considered it worth while to investigate the mechanical possibilities of the light alloys of two somewhat analogous ternary series; namely, of aluminum-magnesium-nickel and of aluminum-magnesium-manganese, to study the effect of variation of composition upon mechanical properties within the aluminum-rich group of the aluminum-magnesium-copper alloys and to compare the mechanical properties of the three ternary series.

1 A. Wilm, Physical-metallurgical investigations of aluminum alloys containing magnesium, Metallurgie, 8, pp. 225, 650; 1911.

2 L. M. Cohn, Duralumin, Zeit. z. Beförderung d. Gewerbefleisses, 89, p. 643; rgro. Also in Electrotechnik u. Maschinenbau, 30, pp. 809, 829; 1912. 
This work was carried out with the cooperation of the Aluminum Co. of America; the alloys were prepared there and most of the mechanical tests were performed by Mr. Waltenberg in the laboratories of the company at New Kensington. The authors wish to express their appreciation of the aid and assistance thus given by the company through E. Blough.

No previous investigation has been made, or at least published, of these three ternary systems, except that dealing with the compositions called duralumin mentioned above. Light alloys of other related alloy series have been prepared and their properties investigated, viz, those of aluminum-copper, ${ }^{3}$ of aluminummanganese, ${ }^{4}$ of aluminum-nickel ${ }^{4,5}$, of aluminum-magnesium, ${ }^{4}$ of aluminum-manganese-copper, ${ }^{6}$ and of aluminum-nickel-copper. ${ }^{3}$

\section{PREPARATION OF THE ALLOYS}

The alloys were prepared by melting standard 99 per cent aluminum ingots with the proper amount of aluminum hardener, consisting of an alloy of aluminum and the magnesium, copper, nickel or manganese depending upon the alloy to be produced. The melts were made in crucibles, attention being given to see that the temperature during melting did not exceed $800^{\circ} \mathrm{C}$, as above this temperature the ingots poured from crucibles are apt to be porous and unsound due to the absorption of gas by the metal. The melting temperature was usually about $700^{\circ} \mathrm{C}$. The molten metal was poured into water-cooled iron molds giving ingots 3.5 by $\mathrm{I} 2$ by 24 inches in dimensions.

These ingots were reheated to from 400 to $450^{\circ} \mathrm{C}$ in a large reheating furnace, sent to the hot rolls, rolled and cross-rolled at these temperatures to a thickness of 0.25 inch. They were then allowed to cool in the air and cold rolled to $0.08 \mathrm{I}$ inch thickness (No. I 2 B. \& S.), annealed at from $400^{\circ}$ to $450^{\circ} \mathrm{C}$, rolled cold to $0.05 \mathrm{I}$ inch thickness (No. I6 B. \& S.), annealed again and finished cold at 0.032 inch thickness.(No. 20 B. \& S.).

Test specimens prepared from these sheets were tested (I) in the cold rolled condition, (2) after annealing at $422^{\circ} \mathrm{C}$, and (3) after heat treatment, consisting of quenching at various temperatures and allowing the quenched specimens to stand or "age"

\footnotetext{
3 H. C. H. Carpenter and C. A. Edwards, Alloys of aluminum and copper, Proc. Inst. Mech. Engineers, p. $57 ;$; 1907 .

4 H. Schirmeister, Investigations of binary aluminum alloys, Stahl u. Eisen, 35, pp. 648, 873; I9I5.

6 A. A. Read and R. H. Greaves, The properties of some aluminum-nickel and of aluminum-nickelcopper alloys, Journ. Inst. Met., 13, p. xoo; rgr5.

6 W. Rosenhain and F. C. Lantsberry, Alloys of copper, aluminum and manganese, Proc. Inst. Mech. Engineers, D. Ixg; I9ro.
} 
for several days before testing. The latter feature of this heat treatment will henceforth be termed "aging," and is necessary in order to develop the highest mechanical properties in the light alloys of the aluminum-copper-magnesium series. It will be noticed that some of the alloys of the latter series were aged at II $0^{\circ} \mathrm{C}$, whereas those of the others were aged only at about room temperature $\left(20^{\circ} \mathrm{C}\right)$.

\section{COMPOSITION OF THE ALLOYS}

It was desired to include in the list of alloys which were to be prepared compositions of each ternary series with the individual percentages of the components varying by intervals of I per cent and with a total combined content of hardening components not exceeding 4.5 per cent, since with a smaller aluminum content these alloys can not be readily rolled into sheet. The actual compositions obtained as determined by chemical analysis are given in Table $\mathrm{I}$. The $\mathrm{B}$ series is that containing manganese, the $\mathrm{C}$ series that containing copper, and the $\mathrm{D}$ series that containing nickel.

\section{MECHANICAL TESTS}

The results of the tests made on the strips cut from the sheets are given in the Tables 2,3 , and 4 .

TABLE 1.-Chemical Composition of Alloys $a$

\begin{tabular}{|c|c|c|c|c|c|c|c|}
\hline Number & $\mathrm{Al}$ & $\mathbf{M g}$ & $\mathrm{Cu}$ & Mn & $\mathrm{Ni}$ & $\mathrm{Fe}$ & $\mathrm{Si}$ \\
\hline B-1... & $\begin{array}{r}\text { Per cent } \\
97.00\end{array}$ & $\begin{array}{r}\text { Per cent } \\
1.15\end{array}$ & $\begin{array}{r}\text { Per cent } \\
0.02\end{array}$ & $\begin{array}{r}\text { Per cent } \\
1.04\end{array}$ & $\begin{array}{c}\text { Per cent } \\
\text { None }\end{array}$ & $\begin{array}{r}\text { Per cent } \\
0.48\end{array}$ & $\begin{array}{r}\text { Per cent } \\
0.31\end{array}$ \\
\hline B-2... & 97.17 & None & .04 & 1.71 & None & .76 & .32 \\
\hline B-3... & 96.14 & 1.09 & .15 & 1.68 & None & .56 & .38 \\
\hline B-4... & 98.02 & None & .08 & 1.07 & None & .44 & .39 \\
\hline B-5... & 95.08 & 2.03 & .08 & 1.68 & None & .76 & .37 \\
\hline B-6:... & 96.86 & 1.44 & .10 & .93 & None & .40 & .27 \\
\hline B-7......... & 96.31 & 1.99 & .03 & .94 & None & .41 & .32 \\
\hline C-1........ & 97.27 & 1.16 & .72 & None & None & .56 & .29 \\
\hline C-2... & 96.69 & 2.37 & .04 & None & None & .62 & .28 \\
\hline C-3....... & 97.15 & None & 2.15 & None & None & .36 & .34 \\
\hline C-4........ & 96.65 & 2.84 & .04 & None & None & .27 & .20 \\
\hline C-5....... & 96.11 & None & 3.19 & None & None & .40 & .30 \\
\hline C-6......... & 96.72 & 2.03 & .72 & None & None & .30 & .23 \\
\hline C-7.......... & 96.62 & 1.00 & 1.80 & None & None & .35 & .23 \\
\hline C-8......... & 96.68 & 1.07 & 1.67 & .02 & None & .33 & .23 \\
\hline C-9......... & 95.98 & 3.50 & .08 & None & None & .26 & .18 \\
\hline C-10......... & 95.83 & 2.95 & .74 & None & None & .27 & .21 \\
\hline C-11......... & 95.51 & 1.26 & 2.58 & .02 & None & .41 & .22 \\
\hline $\mathrm{C}-12 \ldots \ldots \ldots$ & 95.74 & .46 & 3.18 & None & None & .34 & .24 \\
\hline$A-1-12 \ldots \ldots \ldots \ldots \ldots \ldots \ldots$ & 95.48 & .64 & 3.22 & None & None & .39 & .27 \\
\hline
\end{tabular}


TABLE 1-Continued.

\begin{tabular}{|c|c|c|c|c|c|c|c|}
\hline Number & Al & $\mathbf{M g}$ & $\mathrm{Cu}$ & Mn & $\mathrm{Ni}$ & $\mathrm{Fe}$ & Si \\
\hline$E-3 \ldots \ldots \ldots \ldots$ & $\begin{array}{r}\text { Per cent } \\
96.80\end{array}$ & $\begin{array}{r}\text { Per cent } \\
1.06\end{array}$ & $\begin{array}{r}\text { Per cent } \\
1.56\end{array}$ & $\begin{array}{c}\text { Per cent } \\
\text { None }\end{array}$ & $\begin{array}{c}\text { Per cent } \\
\text { None }\end{array}$ & $\begin{array}{r}\text { Per cent } \\
.32\end{array}$ & $\begin{array}{r}\text { Per cent } \\
.26\end{array}$ \\
\hline $\mathrm{D}-1 \ldots \ldots$ & 97.44 & .98 & .02 & None & 1.00 & .36 & .20 \\
\hline $\mathrm{D}-2 \ldots \ldots \ldots \ldots$ & 97.47 & None & .08 & None & 1.76 & .44 & .25 \\
\hline D-3........ & 95.82 & None & .02 & None & 3.40 & .44 & .32 \\
\hline D-4................... & 96.04 & 1.18 & .08 & None & 1.98 & .48 & .24 \\
\hline D-5.................... & 96.70 & 1.84 & .06 & None & .80 & .43 & .17 \\
\hline$D-6 \ldots . .$. & 95.62 & 1.94 & .04 & None & 1.80 & .46 & .14 \\
\hline D-7........ & 95.14 & None & .06 & None & 3.94 & .58 & .28 \\
\hline D-8..................... & 94.65 & .94 & .09 & None & 3.54 & .65 & .13 \\
\hline D-9........ & 95.41 & 2.86 & .06 & None & 1.08 & .39 & .20 \\
\hline
\end{tabular}

TABLE 2.-Mechanical Properties of Alloys of Aluminum-Magnesium-Manganese

\begin{tabular}{|c|c|c|c|c|c|c|c|c|c|c|c|c|}
\hline \multirow[b]{2}{*}{$\underset{\text { ber }}{\text { Num- }}$} & \multicolumn{3}{|c|}{ As rolled } & \multicolumn{3}{|c|}{ - Annealed at $371^{\circ} \mathrm{C}$} & \multicolumn{3}{|c|}{ Annealed at $422^{\circ} \mathrm{C}$} & \multicolumn{3}{|c|}{$\begin{array}{l}\text { Quenched from } 500^{\circ} \mathrm{C} \text {, } \\
\text { aged } 8 \text { days at } 20^{\circ} \mathrm{C}\end{array}$} \\
\hline & $\begin{array}{c}\text { Sclero- } \\
\text { scope } \\
\text { hard-- } \\
\text { ness } a\end{array}$ & $\begin{array}{c}\text { U1timate } \\
\text { tensile } \\
\text { strength }\end{array}$ & $\begin{array}{c}\text { Elonga- } \\
\text { tion } \\
\text { in } 2 \\
\text { inches }\end{array}$ & $\begin{array}{l}\text { Sclero- } \\
\text { scope } \\
\text { hard- } \\
\text { ness } a\end{array}$ & $\begin{array}{c}\text { Ultimate } \\
\text { tensile } \\
\text { strength }\end{array}$ & $\begin{array}{l}\text { Elon- } \\
\text { gation } \\
\text { in } 2 \\
\text { inches }\end{array}$ & $\begin{array}{l}\text { Sclero- } \\
\text { scope } \\
\text { hard- } \\
\text { ness } a\end{array}$ & $\begin{array}{l}\text { Ultimate } \\
\text { tensile } \\
\text { strength }\end{array}$ & $\begin{array}{l}\text { Elonga- } \\
\text { tion } \\
\text { in } 2 \\
\text { inches }\end{array}$ & $\begin{array}{l}\text { Sclero- } \\
\text { scope } \\
\text { hard- } \\
\text { ness } a\end{array}$ & $\begin{array}{c}\text { Ultimate } \\
\text { tensile } \\
\text { strength }\end{array}$ & $\begin{array}{l}\text { Elon- } \\
\text { gation } \\
\text { in } 2 \\
\text { inches }\end{array}$ \\
\hline \multirow[t]{4}{*}{ B-1 } & \multirow[t]{4}{*}{30.0} & $\begin{array}{c}\text { Lbs./in. } .^{2} \\
32400\end{array}$ & $\begin{array}{r}\text { P. ct. } \\
2.0\end{array}$ & \multirow[t]{4}{*}{13.5} & $\begin{array}{c}\text { Lbs./in. } .^{2} \\
25500\end{array}$ & P. ct. & \multirow[t]{4}{*}{11.5} & $\begin{array}{c}\text { Lbs./in. } 2 \\
24700\end{array}$ & P. ct. & \multirow[t]{4}{*}{12.0} & $\begin{array}{c}\text { Lbs./in. }^{2} \\
22300\end{array}$ & $\begin{array}{r}\text { P. ct. } \\
22.0\end{array}$ \\
\hline & & 31600 & 2.0 & & 25300 & ....... & & 24700 & 13.0 & & 23100 & 25.0 \\
\hline & & 32400 & 2.5 & & 25900 & 19.0 & & 23400 & 11.5 & & 22900 & 17.0 \\
\hline & & 31000 & 2.0 & & & & & & & & & \\
\hline \multirow[t]{3}{*}{ B-2 } & \multirow[t]{3}{*}{20.0} & 24000 & 4.5 & 10.5 & 16800 & 26.0 & \multirow[t]{3}{*}{8.5} & 16000 & 30.5 & \multirow[t]{3}{*}{8.5} & 14200 & 36.5 \\
\hline & & 22000 & 3.5 & & 16700 & 24.0 & & 16000 & 35.5 & & 14600 & 36.5 \\
\hline & & 24100 & 4.0 & & 16200 & 31.0 & & 15800 & 30.0 & & 14400 & 35.0 \\
\hline \multirow[t]{3}{*}{ B-3 } & \multirow[t]{3}{*}{32.0} & 34600 & 2.0 & & & & \multirow[t]{3}{*}{14.0} & 26400 & ....... & \multirow[t]{3}{*}{14.0} & 25300 & 18.0 \\
\hline & & 35000 & 2.0 & & & & & 26000 & 14.0 & & 24700 & 17.5 \\
\hline & & 35600 & 2.5 & & & & & 25800 & 12.0 & & 24900 & 20.0 \\
\hline \multirow[t]{7}{*}{ B-4 } & \multirow[t]{7}{*}{20.0} & 21500 & $\ldots$. & & & & \multirow[t]{7}{*}{6.5} & 14300 & 42.0 & \multirow[t]{7}{*}{7.0} & 12800 & 26.0 \\
\hline & & 22500 & 3.0 & & & & & 13700 & 41.0 & & 12900 & 40.0 \\
\hline & & 22100 & 3.0 & & & & & 13300 & 41.0 & & 12300 & 35.0 \\
\hline & & 23500 & 3.0 & & & & & & & & & \\
\hline & & 22900 & 3.0 & & & & & & & & & \\
\hline & & 22300 & 5.5 & & & & & & & & & \\
\hline & & 23500 & 4.0 & & & & & & & & & \\
\hline \multirow[t]{3}{*}{ B-5 } & \multirow[t]{3}{*}{36.0} & 39300 & 2.0 & & & & \multirow[t]{3}{*}{13.5} & 29400 & 20.0 & \multirow[t]{3}{*}{15.0} & 28800 & 16.0 \\
\hline & & 40300 & 2.0 & & & & & 28600 & 16.0 & & 29400 & 15.0 \\
\hline & & 37500 & 2.0 & & & & & 28600 & ....... & & 28000 & $\ldots .$. \\
\hline \multirow[t]{3}{*}{ B-6 } & \multirow[t]{3}{*}{36.5} & 45000 & 3.5 & & & & \multirow[t]{3}{*}{14.0} & 33100 & 13.0 & 15.5 & 34100 & 19.0 \\
\hline & & 42700 & 2.5 & & & & & & & & 33100 & 17.0 \\
\hline & & & & & & & & & & & 32600 & 17.0 \\
\hline B-7 & 35.5 & 37200 & 2.5 & & & & 13.0 & 29600 & 15.5 & 14.0 & 29000 & 19.5 \\
\hline & & 40700 & 4.0 & & & & & 29200 & 17.0 & & 29000 & 19.5 \\
\hline & & & & & & & & & & & 29400 & 18.0 \\
\hline
\end{tabular}

$a$ Taken with magnifying hammer. 
TABLE 3.-Mechanical Properties of Alloys of Aluminum-Magnesium-Copper

\begin{tabular}{|c|c|c|c|c|c|c|c|c|c|}
\hline \multirow[b]{2}{*}{$\underset{\text { ber }}{\text { Num- }}$} & \multicolumn{3}{|c|}{ As rolled } & \multicolumn{3}{|c|}{ Annealed at $422^{\circ} \mathrm{C}$} & \multicolumn{3}{|c|}{$\begin{array}{l}\text { Quenched from } 510^{\circ} \mathrm{C} . \\
\text { a, } 20^{\circ} \mathrm{C} ; \mathrm{b}, 110^{\circ} \mathrm{C}\end{array}$} \\
\hline & $\begin{array}{l}\text { Sclero- } \\
\text { scope } \\
\text { hard- } \\
\text { ness } a\end{array}$ & $\begin{array}{l}\text { Oltimate } \\
\text { tensile } \\
\text { strength }\end{array}$ & $\begin{array}{l}\text { Elonga- } \\
\text { tion in } \\
2 \text { inches }\end{array}$ & $\begin{array}{l}\text { Sclero- } \\
\text { scope } \\
\text { hard- } \\
\text { nessa }\end{array}$ & $\begin{array}{l}\text { Ultimate } \\
\text { tensile } \\
\text { strength }\end{array}$ & $\begin{array}{l}\text { Elonga- } \\
\text { tion in } \\
2 \text { inches }\end{array}$ & $\begin{array}{l}\text { Sclero- } \\
\text { scope } \\
\text { hard- } \\
\text { ness } a\end{array}$ & $\begin{array}{l}\text { Ultimate } \\
\text { tensile } \\
\text { strength }\end{array}$ & $\begin{array}{l}\text { Elonga- } \\
\text { tion in } \\
2 \text { inches }\end{array}$ \\
\hline \multirow[t]{4}{*}{ C- 1} & \multirow[t]{4}{*}{42} & $\begin{array}{r}\text { Lbs./in. }{ }^{2} \\
49000\end{array}$ & $\begin{array}{r}\text { Per cent } \\
2.0\end{array}$ & \multirow[t]{4}{*}{15.5} & $\begin{array}{r}\text { Lbs./in. } 2 \\
33300\end{array}$ & $\begin{array}{l}\text { Per cent } \\
\cdots\end{array}$ & \multirow[t]{2}{*}{17} & \multirow[t]{2}{*}{$\begin{array}{l}\text { Lbs./in. }{ }^{2} \\
\text { a } \begin{cases}38 & 030 \\
37 & 220\end{cases} \end{array}$} & $\begin{array}{r}\text { Per cent } \\
17.0\end{array}$ \\
\hline & & 48400 & 2.5 & & 33100 & 15.0 & & & 16.5 \\
\hline & & 48600 & 2.5 & & 32700 & 14.0 & 27 & $b\left\{\begin{array}{lll}48 & 120\end{array}\right.$ & 16.0 \\
\hline & & 49600 & 2.5 & & & & & D $\begin{cases}47210\end{cases}$ & 18.5 \\
\hline \multirow[t]{4}{*}{ c- 2} & \multirow[t]{4}{*}{19} & 25800 & 4.0 & \multirow[t]{4}{*}{7.5} & 16600 & 35.0 & \multirow[t]{2}{*}{8} & $a \begin{cases}16 & 670\end{cases}$ & 34.0 \\
\hline & & 23600 & 3.0 & & 15900 & 35.0 & & a $\begin{cases}16 & 670\end{cases}$ & 33.0 \\
\hline & & \multirow[t]{2}{*}{23600} & \multirow[t]{2}{*}{3.5} & & \multirow[t]{2}{*}{16100} & \multirow[t]{2}{*}{33.0} & \multirow[t]{2}{*}{8} & $b \begin{cases}16 & 510\end{cases}$ & 28.0 \\
\hline & & & & & & & & 16 510 & 33.0 \\
\hline \multirow[t]{3}{*}{ C- 3} & \multirow[t]{4}{*}{35} & 34900 & 2.5 & \multirow[t]{3}{*}{7.0} & 21600 & 31.0 & 13 & $a\{26350$ & 19.0 \\
\hline & & 35700 & $\cdots \cdots$ & & 21800 & 33.0 & & a $\begin{cases}27690\end{cases}$ & 11.5 \\
\hline & & 34000 & 1.5 & & 22000 & 33.5 & 11 & $b \begin{cases}29 & 420\end{cases}$ & 20.0 \\
\hline$C-4$ & & & & 10.5 & & 180 & 11 & (2) 30060 & 19.5 \\
\hline-4 & 31 & 38400 & 15 & 10.5 & $\begin{array}{rl}29 & 200 \\
59 & 200\end{array}$ & 18.0 & 11 & a $\left\{\begin{array}{l}3000 \\
20700\end{array}\right.$ & 23.0 \\
\hline & & 38600 & 1.5 & & 29200 & 18.0 & & 29700 & 16.5 \\
\hline & & 37200 & 1.5 & & 29400 & 21.0 & 14 & $b \begin{cases}31590 \\
31 & 350\end{cases}$ & 19.0 \\
\hline C- 5 & 34 & 35900 & ..... & 8.0 & 23000 & 30.0 & 14 & $\int 31960$ & 15.5 \\
\hline & & 37500 & 2.5 & & 22400 & 28.5 & & 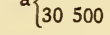 & 14.0 \\
\hline & & 37700 & 2.0 & & 22800 & 32.5 & 14 & b $\begin{cases}30910\end{cases}$ & 19.0 \\
\hline C- 6 & 38 & 35300 & 1.0 & 13.0 & 30500 & ....... & 15 & $\left\{\begin{array}{l}33970 \\
33370\end{array}\right.$ & 17.0 \\
\hline & & 38500 & 0.5 & & 29900 & 18.5 & & a $\left\{\begin{array}{l}33950 \\
\text { a }\end{array}\right.$ & 23.5 \\
\hline & & 38100 & 1.5 & & 30800 & 16.0 & 26 & b) $\int^{43190}$ & 18.5 \\
\hline & & & & & & & & $\begin{cases}43560 \\
0\end{cases}$ & 18.0 \\
\hline$c-7$ & 44 & 44200 & 2.0 & 17.0 & 35300 & 26.0 & 24 & $a\left\{\begin{array}{l}45650 \\
0\end{array}\right.$ & 18.5 \\
\hline & & 45500 & 2.0 & & 34600 & 25.5 & & d $\left\{\begin{array}{l}45740 \\
\text {. }\end{array}\right.$ & 19.5 \\
\hline & & 45300 & ...... & & 34800 & 25.0 & 35 & b $\left\{\begin{array}{l}53970 \\
0\end{array}\right.$ & 20.0 \\
\hline & & & & & & & & {$\left[\begin{array}{ll}52 & 250\end{array}\right.$} & ..... \\
\hline$C-8$ & 38 & 38100 & 1.5 & 12.5 & 28500 & 18.5 & & & \\
\hline & & 38100 & ..... & & 29100 & 18.5 & & & \\
\hline C- 9 & 38 & 41200 & 1.5 & 12.0 & 31600 & 17.5 & 13 & $\int 29120$ & 21.0 \\
\hline & & 43200 & 1.5 & & 31200 & 17.5 & & a $\left\{\begin{array}{l}29500 \\
20\end{array}\right.$ & 22.0 \\
\hline & & 41200 & 1.5 & & 30500 & ...... & 14 & b $\begin{cases}30 & 270\end{cases}$ & 23.0 \\
\hline & & & & & & & & $\begin{cases}30 & 270\end{cases}$ & 22.0 \\
\hline $\mathrm{C}-10$ & 45 & 44800 & 1.5 & 12.0 & 30600 & 19.0 & 14 & $\int 37430$ & 24.5 \\
\hline & & 44600 & 1.5 & & 30200 & 19.0 & & ${ }^{a}\{37630$ & 21.5 \\
\hline & & 47500 & 1.5 & & 30200 & 17.0 & 26 & b $\{47690$ & 21.5 \\
\hline & & & & & & & & D $\left\{\begin{array}{l}47690 \\
0\end{array}\right.$ & 22.5 \\
\hline$C-11$ & 50 & 56700 & 2.0 & 15.5 & 34900 & 20.5 & 29.5 & $a \begin{cases}51520\end{cases}$ & 21.0 \\
\hline & & 52900 & 1.5 & & 36000 & 24.0 & & a $\begin{cases}50 & 870\end{cases}$ & 24.0 \\
\hline & & 58400 & 2.0 & & & & 34 & b $\begin{cases}54740 & \end{cases}$ & 23.0 \\
\hline $\mathrm{C}-12$ & 31 & 38900 & 5.0 & 7.5 & 23100 & 24.0 & $25-28$ & {$\left[\begin{array}{ll}55 & 590 \\
42 & 370\end{array}\right.$} & $\begin{array}{l}20.0 \\
14.5\end{array}$ \\
\hline & & 38600 & 5.0 & & 23000 & 24.0 & & ${ }^{a} \begin{cases}39 & 340\end{cases}$ & 16.5 \\
\hline & & & & & & & 26 & b $\begin{cases}49 & 230\end{cases}$ & 26.5 \\
\hline & & & & & & & & l49 830 & 25.5 \\
\hline
\end{tabular}

a Taken with magnifying hammer. 
TABLE 4.-Mechanical Properties of Alloys of Aluminum-Magnesium-Nickel

\begin{tabular}{|c|c|c|c|c|c|c|c|c|c|c|}
\hline \multirow[b]{2}{*}{ Ivum- } & \multicolumn{4}{|c|}{ As rolled } & \multicolumn{3}{|c|}{ Annealed at $500^{\circ} \mathrm{C}$} & \multicolumn{3}{|c|}{$\begin{array}{l}\text { Quenched from } 500^{\circ} \mathrm{C} \text {. } \\
\text { Aged } 20 \text { days, at } 20^{\circ} \mathrm{C}\end{array}$} \\
\hline & $\begin{array}{l}\text { Sclero- } \\
\text { scope } \\
\text { hard- } \\
\text { ness } a\end{array}$ & $\begin{array}{l}\text { Ultimate } \\
\text { tensile } \\
\text { strength }\end{array}$ & $\begin{array}{l}\text { Yield } \\
\text { point }\end{array}$ & $\begin{array}{l}\text { Elonga- } \\
\text { tion in } \\
2 \text { inches }\end{array}$ & $\begin{array}{l}\text { Sclero- } \\
\text { scope } \\
\text { hard- } \\
\text { ness } a\end{array}$ & $\begin{array}{c}\text { Ultimate } \\
\text { tensile } \\
\text { strength }\end{array}$ & $\begin{array}{l}\text { Elonga- } \\
\text { tion in } \\
2 \text { inches }\end{array}$ & $\begin{array}{l}\text { Sclero- } \\
\text { scope } \\
\text { hard- } \\
\text { ness } a\end{array}$ & $\begin{array}{c}\text { Ultimate } \\
\text { tensile } \\
\text { strength }\end{array}$ & $\begin{array}{l}\text { Elonga- } \\
\text { tion in } \\
2 \text { inches }\end{array}$ \\
\hline \multirow{2}{*}{ D-1 } & 23 & $\begin{array}{r}\text { Lbs./in. } 2 \\
23800\end{array}$ & . & \begin{tabular}{|} 
Per cent \\
3.0
\end{tabular} & 7 & $\begin{array}{c}\text { Lbs./in. }{ }^{2} \\
17800\end{array}$ & $\begin{array}{r}\text { Per cent } \\
15.0\end{array}$ & 15 & $\begin{array}{l}\text { Lbs./in. } 2 \\
27700\end{array}$ & $\begin{array}{r}\text { Per cent } \\
18.0\end{array}$ \\
\hline & & 25500 & 21000 & 3.5 & 7 & 17900 & 18.5 & 14 & 26200 & 21.0 \\
\hline \multirow[t]{2}{*}{ D-2 } & 22 & 24900 & 17500 & 2.5 & 7 & 18000 & 29.5 & 7.5 & 18600 & 28.5 \\
\hline & & 25200 & 18000 & 2.5 & 6.5 & 17700 & 24.0 & 7 & 18600 & 27.0 \\
\hline \multirow[t]{2}{*}{ D-3 } & 27 & 32100 & …...... & 3.0 & 8 & 21600 & 27.0 & 10 & 21600 & 24.0 \\
\hline & & 32600 & …..... & 3.5 & 8 & 20600 & 29.0 & 9 & 21600 & 20.0 \\
\hline \multirow[t]{2}{*}{ D-4 } & 27.5 & 29900 & 25500 & 2.0 & 9.5 & 20900 & 20.0 & 13 & 27900 & 18.0 \\
\hline & & 29300 & 25500 & 2.5 & 9.5 & 21100 & 20.5 & 14 & 27100 & 17.0 \\
\hline \multirow[t]{2}{*}{ D-5 } & 29 & 33000 & 30000 & 4.0 & 10 & 24200 & 21.0 & 12 & 28100 & 19.0 \\
\hline & & 34300 & 29000 & 4.5 & 11 & 21100 & 16.5 & 12.5 & 27600 & 18.5 \\
\hline \multirow[t]{2}{*}{ D-6 } & 30 & 36900 & 31000 & 1.5 & 11 & 25200 & 18.0 & 14 & 29900 & 20.0 \\
\hline & & 35200 & 31000 & 1.0 & 12 & 25400 & 18.5 & 13.5 & 29100 & 21.5 \\
\hline \multirow[t]{2}{*}{ D-7 } & 24 & 29200 & 21000 & 3.0 & 10 & 20200 & 23.0 & 9 & 22700 & 21.5 \\
\hline & & 29300 & 21000 & 3.0 & 10 & 20200 & 24.5 & 10 & 22400 & 21.0 \\
\hline \multirow[t]{2}{*}{ D-8 } & 28 & 33800 & 28500 & 2.5 & 10.5 & 21400 & 19.0 & 16 & 31000 & 15.5 \\
\hline & & 34100 & 29000 & 1.5 & 11 & 22600 & 19.0 & 15 & 31500 & 15.0 \\
\hline \multirow[t]{2}{*}{ D-9 } & 32 & 42000 & 35000 & 3.0 & 13 & 28900 & ..... & 14 & 32200 & 18.0 \\
\hline & & 40600 & 34500 & 3.0 & 13.5 & 28800 & & 15 & 31800 & 16.0 \\
\hline
\end{tabular}

$a$ Taken with magnifying hammer.

From a study of these tables several facts are apparent.

The alloys of aluminum-manganese and of aluminum-manganesemagnesium are not improved by heat-treatment of the type used for duralumin. The alloys of aluminum-nickel alone are also not appreciably affected by this heat-treatment (see Nos. D-2, D-3, and $\mathrm{D}-7$ ), but in those alloys in which both nickel and magnesium are present the heat-treated specimens are harder and stronger than the annealed ones.

Within the $\mathrm{C}$ series it is noticed that alloys containing magnesium but no copper are not improved by heat-treatment; alloys containing copper but no magnesium are moderately affected; the greatest increases in hardness and strength are found in the heattreated specimens of alloys containing both copper and magnesium.

Table 5 gives a survey of the percentage increase of strength of the heat-treated over the annealed specimens of the same composition.

In the annealed condition and for equal additions, either with or without magnesium, copper seems to confer the greatest hardening effect, next manganese, and then nickel. This is shown in the comparisons of Table 6 . 
At about 3 per cent, magnesium alone appears to exert a greater hardening effect than the same percentage of the other metals, but at about 2 per cent exerts a lesser effect than that of copper.

TABLE 5.-Percentage Increase of Tensile Strength of Heat-treated Specimens over Annealed Ones of the Same Composition

\begin{tabular}{|c|c|c|c|}
\hline Alloys containing- & $\begin{array}{l}\text { Average } \\
\text { increase }\end{array}$ & Alloys containing - & $\begin{array}{l}\text { Average } \\
\text { increase }\end{array}$ \\
\hline $\begin{array}{l}\text { Manganese, no magnesium B-2 (-9 per } \\
\text { cent) B-4 ( }-7 \text { per cent)............. } \\
\text { Manganese and magnesium B-1 }(-4 \text { per } \\
\text { cent) B-3 ( }-5 \text { per cent) B-5 (0) B-6 (0) } \\
\text { B-7 ( }-2 \text { per cent)................ } \\
\text { Nickel, no magnesium D-2 (3 per cent) } \\
\text { D-3 (3 per cent) D-7 (12 per cent)..... } \\
\text { Nickel and magnesium D-1 (40 per cent) } \\
\text { D-4 (31 per cent) D-5 (24 per cent) D-6 } \\
\text { (17 per cent) D-8 (41 per cent) D-9 ( } 11 \\
\text { per cent)........................... }\end{array}$ & $\begin{array}{r}\text { Per cent } \\
-8 \\
-3 \\
6\end{array}$ & 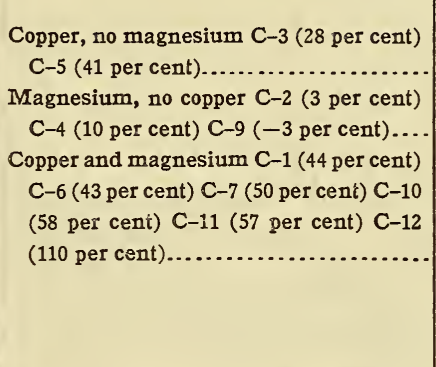 & $\begin{array}{r}\text { Per cent } \\
34 \\
3\end{array}$ \\
\hline
\end{tabular}

TABLE 6.-Comparison of the Hardening or Strengthening Effect of Copper, of Nickel, of Manganese, and of Magnesium on the Annealed Specimens

\begin{tabular}{|c|c|c|c|}
\hline Number & Composition & $\begin{array}{l}\text { Tensile } \\
\text { strength }\end{array}$ & Elongation \\
\hline C-3........................ & 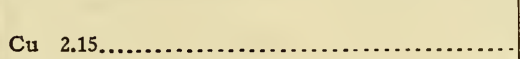 & $\begin{array}{c}\text { Lbs./in. } 2 \\
21800\end{array}$ & 32 \\
\hline B-2................... & Mn $1.71 \ldots \ldots \ldots \ldots \ldots \ldots \ldots \ldots \ldots \ldots \ldots \ldots \ldots \ldots \ldots$ & 16000 & 32 \\
\hline D-2.............. & 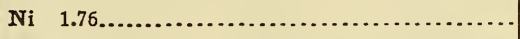 & 17800 & 27 \\
\hline C-2............... & 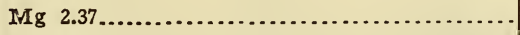 & 16000 & 35 \\
\hline $\mathrm{C}-5 \ldots \ldots$ & 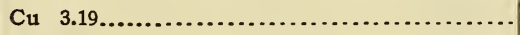 & 22800 & 30 \\
\hline D-3....... & 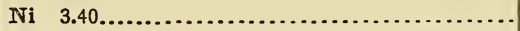 & 21100 & 28 \\
\hline C $-4 \ldots$. & $\mathrm{Mg} 2.84 \ldots \ldots \ldots \ldots \ldots \ldots \ldots$ & 29000 & 19 \\
\hline $\mathrm{C}-1 \ldots .$. & 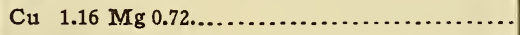 & 33000 & 15 \\
\hline B-1...... & Mn $1.04 \mathrm{Mg} 1.15 \ldots \ldots \ldots \ldots \ldots \ldots \ldots \ldots \ldots \ldots \ldots$ & 24000 & 12 \\
\hline D-1.... & 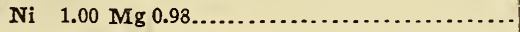 & 17700 & 17 \\
\hline C-7...... & 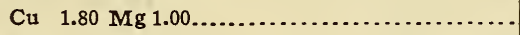 & 35000 & 25 \\
\hline B-3.................... & 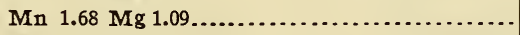 & 26000 & 13 \\
\hline D-4....... & 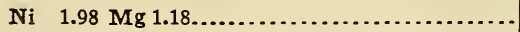 & 21000 & 20 \\
\hline C-6...... & 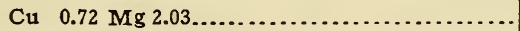 & 30000 & 17 \\
\hline B-5... & $\mathrm{Mn} 1.68 \mathrm{Mg} 2.03 \ldots \ldots \ldots \ldots$ & 29000 & 18 \\
\hline D-6................. & 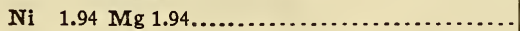 & 25000 & 18 \\
\hline
\end{tabular}

\section{CORROSION TEST}

The resistance of these alloys to corrosion was determined by the salt spray test. This test consists of exposing the samples to a continuous fog of salt water, produced by atomizing a 20 per cent solution of salt (sodium chloride) in water. ${ }^{7}$

\footnotetext{
${ }^{7}$ A. N. Finn. Method of Making Salt Spray Test, Proc. Am. Soc. Test. Mats., XVIII, Part r, p. 237;
} 1918 . 
Although this test is not considered as entirely satisfactory, it is thought that the results produced represent with a fair degree of accuracy the results obtained in actual service, especially under marine conditions.

The alloys were subjected to the salt spray test for two periods of one month each, and were examined at the end of each period to determine the relative amount of corrosion. This was estimated by appearance only as it is practically impossible to determine it by loss in weight on account of the adherence of the aluminum rust and the lack of a satisfactory reagent to remove the rust without affecting the metallic aluminum.

The test pieces were 4 by 2 by 0.03 inches and included each series of alloys treated as follows: (I) as rolled (marked $\mathrm{B}-4$, etc.), (2) quenched from $520^{\circ} \mathrm{C}$ into water at $16^{\circ} \mathrm{C}$ (marked $\mathrm{B}-4-\mathrm{A}$, etc.), (3) quenched from $520^{\circ} \mathrm{C}$ into water at $100^{\circ} \mathrm{C}$ (marked $\mathrm{C}-2-\mathrm{B}$, etc.), (4) annealed at $45^{\circ} \mathrm{C}$ and cooled slowly (marked B-4-C, etc.). Specimens of commercial sheet aluminum, (I) as rolled marked (AI), (2) annealed at $450^{\circ} \mathrm{C}(\mathrm{Al}-\mathrm{A}),(3)$ annealed at $500^{\circ} \mathrm{C}(\mathrm{Al}-\mathrm{B})$, and (4) quenched from $500^{\circ} \mathrm{C}(\mathrm{Al}-\mathrm{C})$ were tested in the same way.

After one month's exposure to the salt spray there was a marked difference in the appearance of the various rolled alloys. B-4 and $\mathrm{B}-5$ as rolled, annealed, and quenched were only slightly corroded and appeared better than the remaining alloys. The annealed specimens, $\mathrm{B}-4-\mathrm{C}$, and $\mathrm{B}-5-\mathrm{C}$ were corroded more than the rolled or the quenched specimens of these alloys.

Specimens of D-3, D-6, and D-8, rolled, annealed, and quenched were not quite as good as the B series. The specimens as rolled were corroded a little more than the annealed or quenched specimens.

The rolled specimens of the $\mathrm{C}$ series $(\mathrm{C}-2, \mathrm{C}-5, \mathrm{C}-8$, etc.), with the exception of $\mathrm{C}-2$ were badly corroded. $\mathrm{C}-2$, as rolled, annealed, and quenched, C-II annealed and quenched, and the quenched specimens of the remainder of this series compared favorably with $\mathrm{B}-4$ and $\mathrm{B}-5$.

Figs. I, 2, and 3 show the appearance of some of the specimens after one month's exposure.

In the following table the alloys are grouped according to their resistance to corrosion, as indicated by their appearance at the end of the second month, group I being the most resistant and group IV the least resistant. 


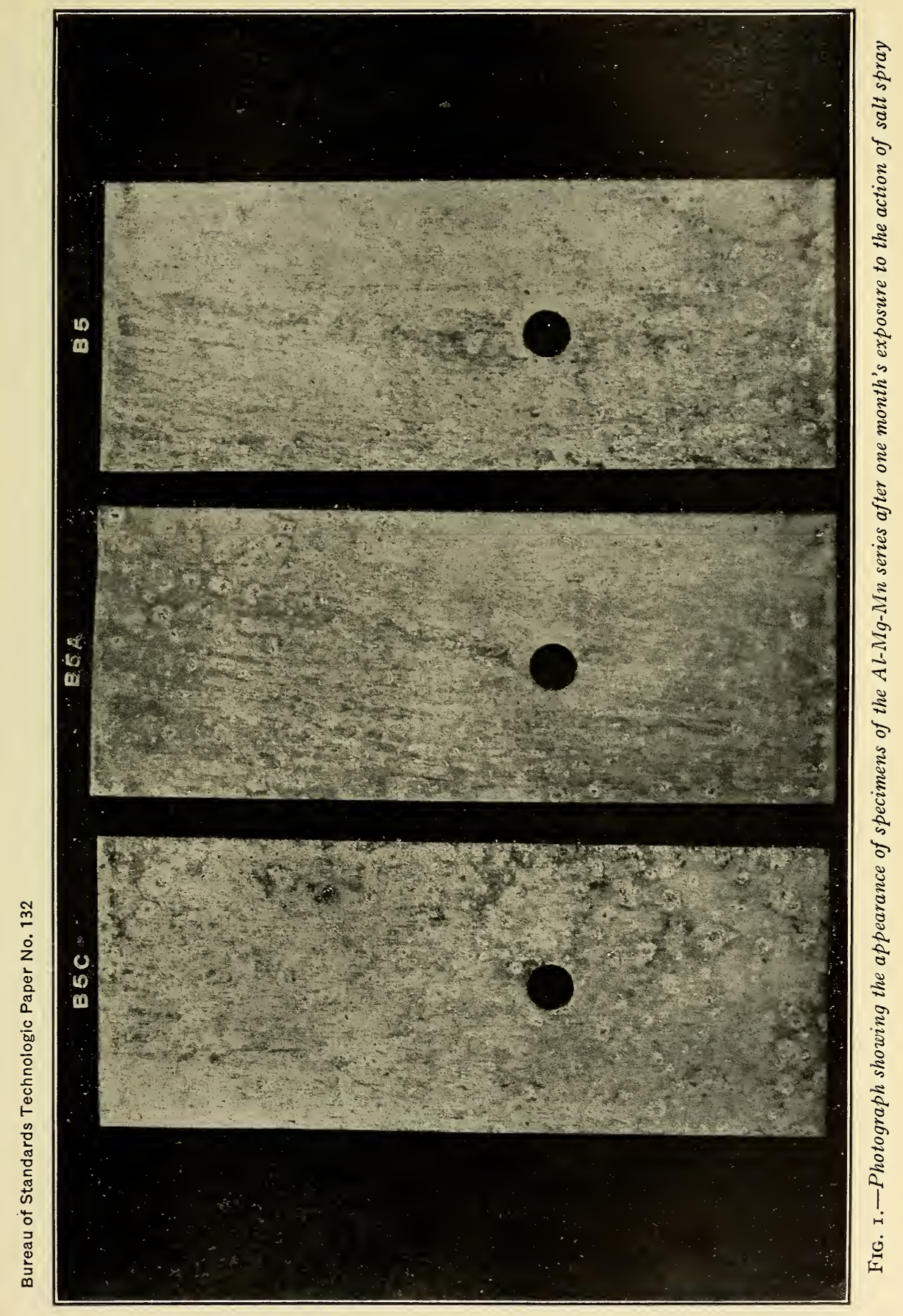




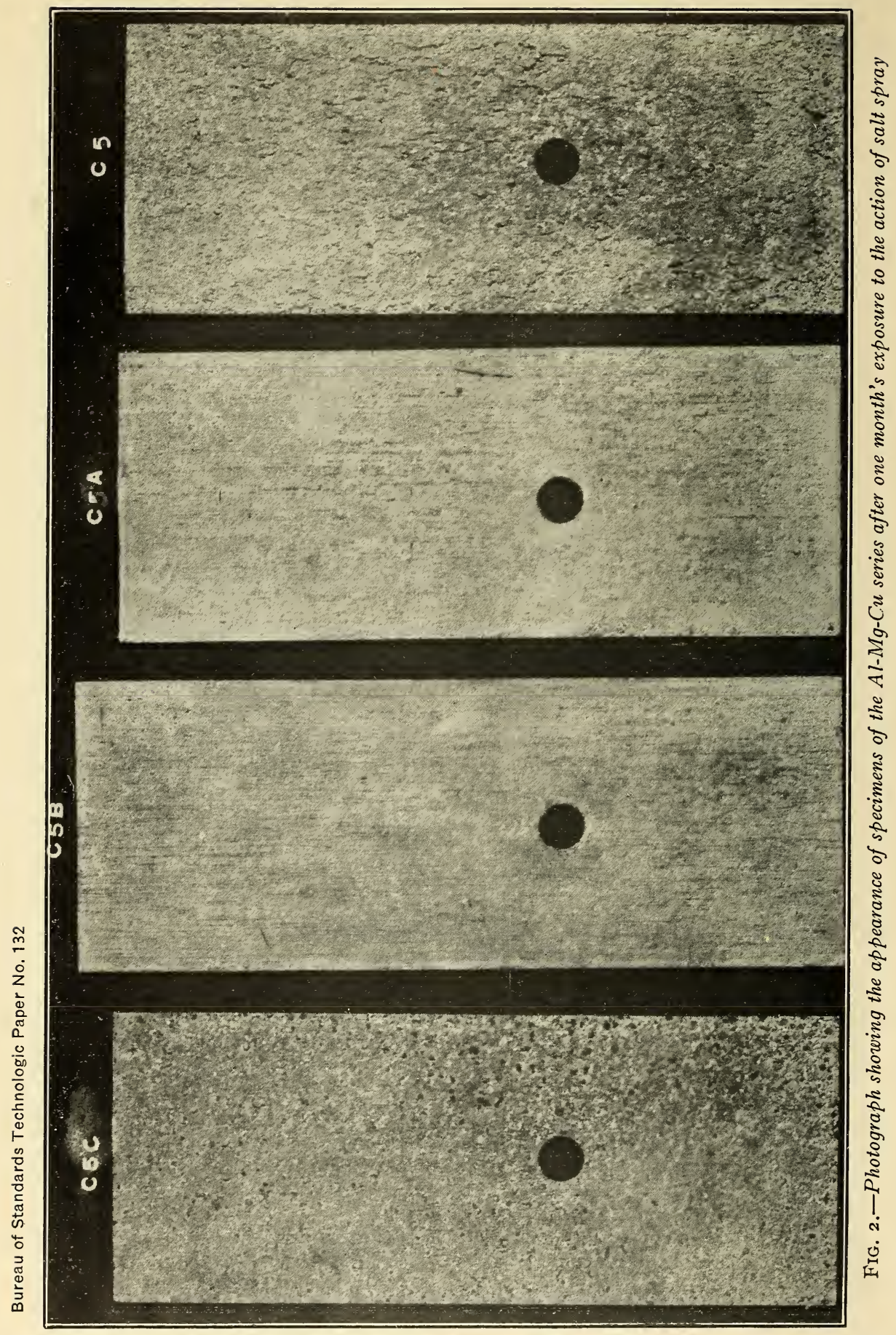


Arrangement of Specimens in the Order of Their Resistance to Corrosion

\begin{tabular}{|l|l|l|l|l|}
\hline \multicolumn{1}{|c|}{ I } & \multicolumn{2}{|c|}{ II } & \multicolumn{2}{|c|}{ III } \\
\cline { 2 - 5 } B-5-A & C-11-B & B-5-C & C-11-C & C-11 \\
B-4-A & C-11-A & B-4-C & C-2 & C-8 \\
B-5 & C-5-B & B-4 & C-8-C & C-5 \\
C-8-B & C-5-A & C-2-B & D-3-A & C-12 \\
C-8-A & C-12-A & D-6-C & C-2-C & C-5-C \\
& C-12-B & D-6-A & C-2-A & C-12-C \\
& & D-6 & D-3 & A1 \\
& & A1-A & D-8 & \\
& & A1-B & D-3-C & \\
& & A1-C & D-8-A & \\
& & & D-8-C & \\
\hline
\end{tabular}

It must be stated that there was not the distinct difference in corrosive effect that might be inferred from the above classification, but the difference was sometimes very small or negligible between pieces in the same group, and group I merges with group II, but the difference between groups II and III, and between III and IV is very definite.

The order in which the 39 samples listed above are grouped is based on the opinion of two observers working independently of each other and it is noteworthy that the results of their observations were in almost complete agreement. In no case was any sample placed by either observer in different groups than that indicated above, and in only a few cases did the indicated order differ.

The following table gives, in condensed form, a summary of the corrosion tests and shows at a glance the relative resistance to corrosion of the alloys studied and the effect produced by quenching and annealing the rolled alloys.

The figures in the table indicate the group in which a particular alloy was classed with respect to its resistance to corrosion. (See previous table.)

Relative Corrosion

\begin{tabular}{|c|c|c|c|c|c|c|c|c|}
\hline & B-4 & B-5 & $\mathrm{C}-2$ & $\mathrm{C}-5$ & C-8, C-11, & $D-3$ & $\begin{array}{l}\text { D-6, } \\
\text { D-8 }\end{array}$ & A1 \\
\hline Alloying metals..................... & Mn & $\begin{array}{l}\mathrm{Mn}- \\
\mathrm{Mg}\end{array}$ & $\mathbf{M g}$ & $\mathrm{Cu}$ & $\mathrm{Cu}-\mathrm{Mg}$ & Ni & Ni-Mg & \\
\hline As rolled............................. & 3 & 1 & 3 & 4 & 4 & 3 & 3 & 4 \\
\hline Quenched $520^{\circ}$ to $16^{\circ} \mathrm{C}$. (A)... & 1 & 1 & 3 & 2 & 1 and 2 & 3 & 3 & 3 \\
\hline Quenched $520^{\circ}$ to $100^{\circ} \mathrm{C}$. (B).. & (a) & (a) & 3 & 2 & 1 and 2 & (a) & (a) & (a) \\
\hline Annealed at $450^{\circ} \mathrm{C}$. (C)......... & 3 & 3 & 3 & 4 & 3 and 4 & 3 & 3 & 3 \\
\hline
\end{tabular}

$a$ No samples tested. 
Consideration of this table indicates clearly that a decided difference in resistance to corrosion may be produced by quenching some aluminum alloys, a less marked difference is produced by annealing, and with some alloys no apparent difference is produced.

The following conclusions are drawn from examination of this table: (I) If any change is produced by quenching, it improves the resistance of the metal to corrosion, (2) the magnesium, nickel, and nickel-magnesium alloys have about the same resistance to corrosion regardless of treatment, (3) annealing improves somewhat the resistance to corrosion of the copper-magnesium alloys and reduces the resistance of the manganese-magnesium alloy, (4) quenching produces the greatest effects in the copper, coppermagnesium, and manganese alloys, (5) commercial aluminum as hard rolled does not resist corrosion satisfactorily and the sample tested was almost completely disintegrated at the end of the test, showing characteristic exfoliation. Annealing or quenching materially improves aluminum, but it is not equal to some of the alloys.

\section{SUMMARY}

Light aluminum alloys of several compositions belonging to each of the three ternary series, aluminum-magnesium-copper, aluminum-magnesium-manganese, and aluminum-magnesiumnickel, were rolled out into sheet and tested in tension as coldrolled, after annealing, and after heat treatment, consisting of quenching from about $500^{\circ} \mathrm{C}$ and aging at ordinary temperature.

The alloys of the aluminum-magnesium-copper series were superior in all conditions to those of the other series in respect to tensile properties.

The tensile properties of the aluminum-magnesium-copper series may be much improved by an appropriate heat treatment. The alloys of the aluminum-magnesium-nickel series are also improved by heat treatment, but not in the same degree as the former series. The alloys of the aluminum-magnesium-manganese series are not improved by heat treatment.

Samples of representative compositions of each series were exposed to corrosion in the salt-spray test, and the appearance of the samples observed after one and after two months' exposure to the action of the salt spray.

The alloys of the aluminum-magnesium-manganese series resisted corrosion in general better than those of the other series, and this agrees with other experience in the corrosion of such 


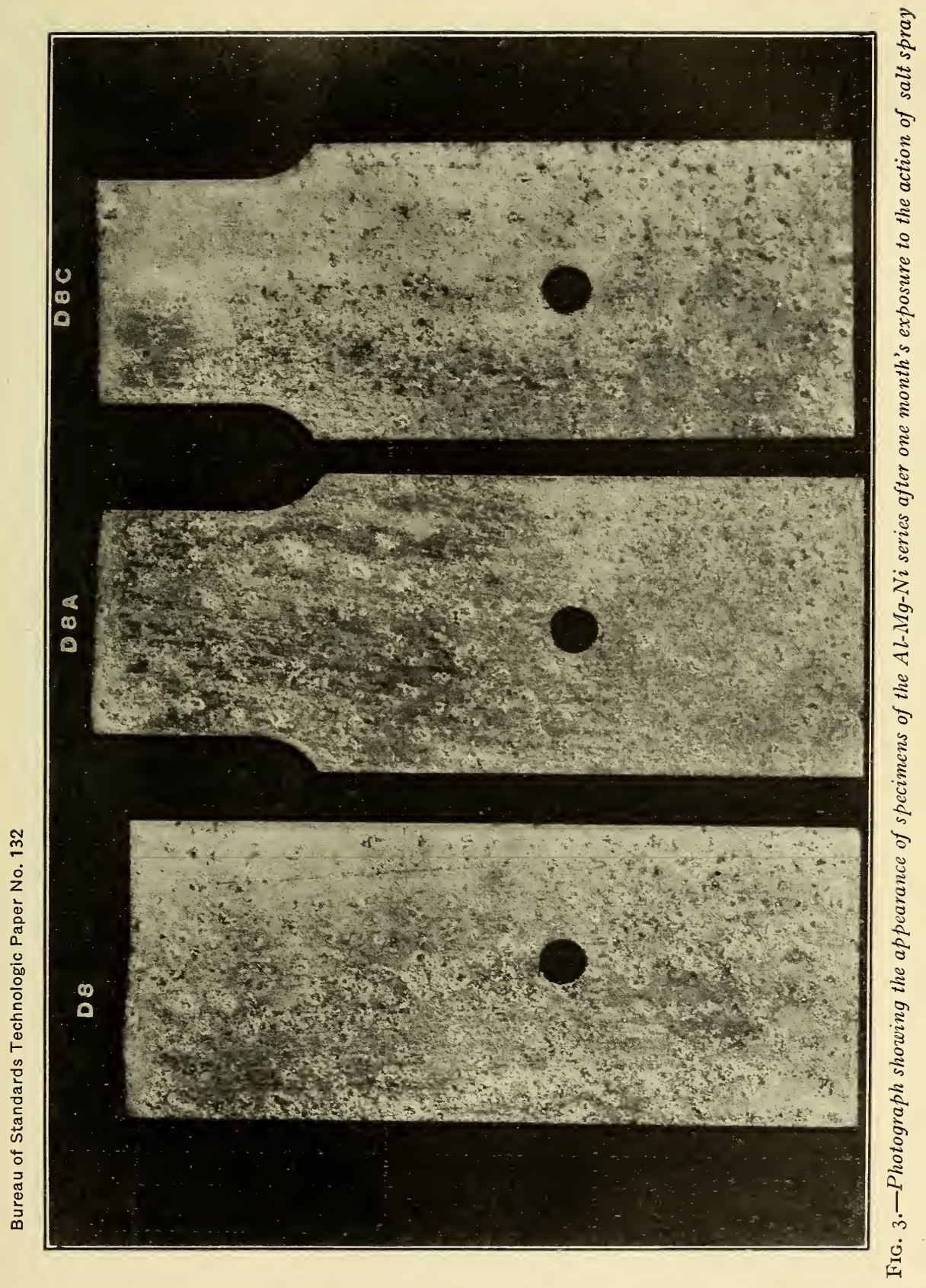



alloys. The heat-treated specimens of the aluminum-magnesiumcopper series were, however, but little inferior to those of the manganese series in their resistance to corrosion; the annealed and the cold-rolled samples of that series were the least resistant to corrosion of any of the alloys tested. Hard-rolled commercial aluminums corroded much more than any of the alloys. Annealed aluminum was more resistant to corrosion than the hard-rolled aluminum, but did not compare favorably with most of the alloys.

WASHINGTON, February 27, I919. 